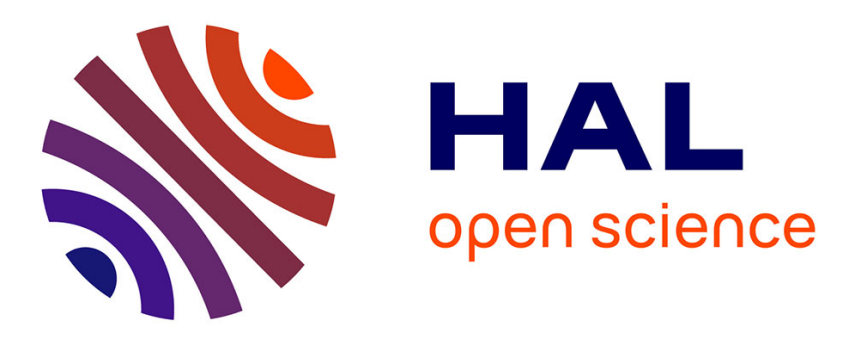

\title{
Instability of hollow polymeric microspheres upon swelling
}

\author{
V. A. Eremeyev, A. B. Freidin, V. N. Pavlyuchenko, S. S. Ivanchev
}

\section{To cite this version:}

V. A. Eremeyev, A. B. Freidin, V. N. Pavlyuchenko, S. S. Ivanchev. Instability of hollow polymeric microspheres upon swelling. Doklady Physics, 2007, 52 (1), pp.37-40. hal-00830635

\section{HAL Id: hal-00830635 \\ https://hal.science/hal-00830635}

Submitted on 5 Jun 2013

HAL is a multi-disciplinary open access archive for the deposit and dissemination of scientific research documents, whether they are published or not. The documents may come from teaching and research institutions in France or abroad, or from public or private research centers.
L'archive ouverte pluridisciplinaire HAL, est destinée au dépôt et à la diffusion de documents scientifiques de niveau recherche, publiés ou non, émanant des établissements d'enseignement et de recherche français ou étrangers, des laboratoires publics ou privés. 


\title{
Instability of Hollow Polymeric Microspheres upon Swelling
}

\author{
V. A. Eremeyev ${ }^{a}$, A. B. Freidin ${ }^{b}$, \\ V. N. Pavlyuchenko ${ }^{a}$, and Corresponding Member of the RAS S. S. Ivanchev ${ }^{c}$
}

Hollow polymeric microspheres of submicron dimensions are widely used in modern technology [1]. Since such microspheres possess a high light scattering power, they are used, for example, in the paper industry as white pigments and in the production of paintwork materials. The light is most effectively scattered by microspheres with diameters about $400-500 \mathrm{~nm}$ and wall thicknesses about $50 \mathrm{~nm}$. A promising technology for manufacturing such microspheres is based on aqueous emulsion polymerization reactions [1]. As a rule, the technological process consists of a large number of stages and involves the isolation of intermediate products. Recently, we have developed $[2,3]$ a simplified "one-pot" technology, according to which the process is carried out in a single reactor and involves only three successive stages: (i) the production of a particle core on the basis of a carboxyl-containing copolymer; (ii) the formation of a polymeric shell around the nucleus; and (iii) the neutralization of carboxyl groups of the core. The latter process is performed using a base at a temperature above the glass transition temperature of the shell polymer and leads to the ionization of carboxyl groups, their hydration, swelling of the core copolymer, and expansion of the shell. As a result, water-filled cavities are formed. On cooling, the polymer of the shell transforms from a highly elastic state into a glassy state. Drying of the particles is accompanied by the diffusion removal of water from the cavities. In the first two stages, chemical processes are most

\footnotetext{
"Southern Scientific Center, Russian Academy of Sciences, Rostov-on-Don, 344090 Russia

${ }^{b}$ Institute for Problems in Mechanical Engineering, Russian Academy of Sciences, St. Petersburg, 199178 Russia

${ }^{c}$ Boreskov Institute of Catalysis (St. Petersburg Branch), Siberian Division, Russian Academy of Sciences, St. Petersburg, 197198 Russia

e-mail:eremeyev@math.rsu.ru
}

significant, whereas, at the third stage, it is also necessary to take into account considerable deformations of the polymer shell, since the hollow volume can increase due to expansion by a factor of 10 compared to the initial core size. Thus, from the standpoint of mechanics, at the third stage we deal with the expansion of a nonlinearly elastic thick-walled shell made of a highly elastic material. Here, the volume of liquid in the hollow acts as a parameter of loading. One of the serious problems that arises in realizing the technological process described above is that, during the formation of cavities in the initial particles, the spherical shape of the shell can be distorted. Previously [1], in order to explain this phenomenon, some assumptions were made concerning the effect of residual stresses after water removal from the hollow of a particle, the rupture of the shell material at the stage of expansion, etc. However, the factors actually responsible for the distortions of the regular spherical shape of the particles are still not reliably established. It is also unclear when the particles with a defect structure appear at the third stage (during expansion or upon cooling, i.e., during the polymer transition from a highly elastic state to a glassy state).

In this paper, it is shown that the loss of sphericity can be associated with instability of the shell in the course of expansion. We investigated the loss of local stability in a thick-walled spherical shell made of a highly elastic incompressible material with respect to axisymmetric perturbations retaining the hollow volume. The shell is assumed to be loaded by the internal hydrostatic pressure produced by the incompressible liquid absorbed by the hydrophilic core.

In the framework of the three-dimensional nonlinear theory of elasticity, the instability under the conditions of extension was investigated by Zubov et al. [4-6]. In particular, the investigation [4] of the instability of an elastic beam on tension showed that the bifurcation of equilibrium takes place only in the case when the deformation diagram (stress-strain curve) has a descending region. A similar situation is observed during expansion 


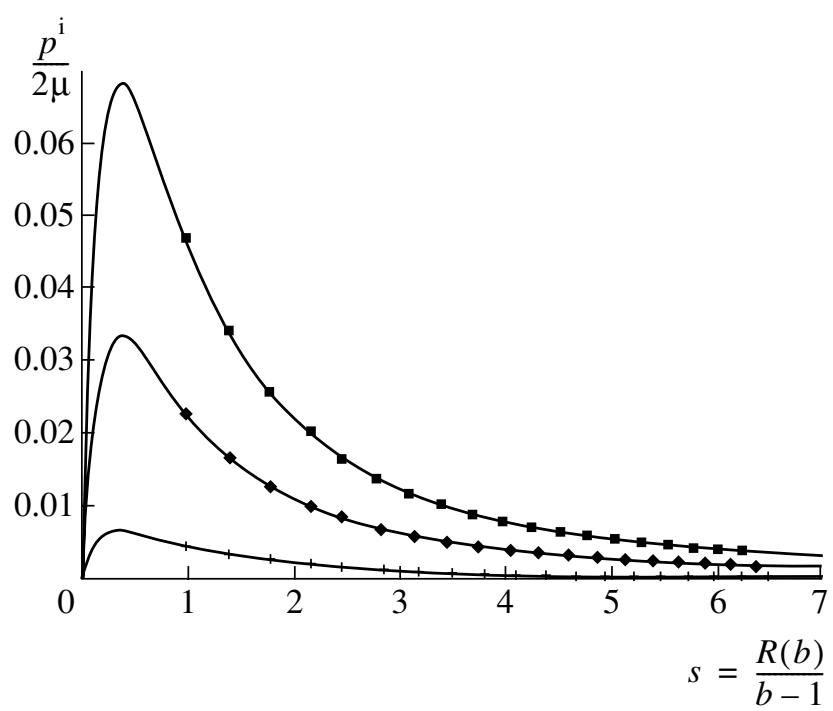

Fig. 1. Diagrams of expansion for various shell thicknesses $h=0.1,0.05$, and 0.01 and the bifurcation points corresponding to various mode numbers $n$.

of a nonlinearly elastic cylinder [5, 6]. Various problems of the mechanics of materials having descending regions on the deformation diagram were discussed in $[7,8]$. Using methods of the theory of shells, the problem of stability of an expanded elastic sphere was earlier solved in [9], where the forms of the loss of sphericity of the shell after expansion were also found. Instability of a spherical shell during expansion was also considered in [10], where a mechanism of the disturbance of the symmetric spherical shape due to the instability was associated with the appearance of nonsymmetric solutions at rather high loads. The existence of analogous nonsymmetric solutions to the problem of equilibrium of a symmetrically loaded round plate under the conditions of increasing transverse load was demonstrated by Morozov [11]. The maintenance of the optimum spherical shape of a polymer shell during expansion offers an example of the problem of optimal design for elastic bodies [12].

Initial state. The basic relationship for a highly elastic material was chosen in the form described by the Bartenev-Khazanovich model [13]

$$
\begin{gathered}
\mathbf{D}=2 \mu \mathbf{A}-p \mathbf{C}^{-T}, \\
\mathbf{C}=\operatorname{grad} \mathbf{R}, \quad \mathbf{A}=\mathbf{U}^{-1} \cdot \mathbf{C}, \quad \mathbf{U}=\left(\mathbf{C} \cdot \mathbf{C}^{T}\right)^{1 / 2}, \\
\operatorname{det} \mathbf{C}=1
\end{gathered}
$$

where $\mathbf{D}$ is the Piola stress tensor, $\mathbf{C}$ is the deformation gradient, $\mathbf{R}$ is the radius vector of a point of a body in the deformed state, $p$ is the response "pressure" (which is the unknown function of the coordinates and is independent of deformation), and $\mu$ is the elastic constant (playing the role of the shear modulus for small deformations).
The radially symmetric deformation of an incompressible body is described by the following formulas [13]:

$$
R=\sqrt[3]{r^{3}-c}, \quad \Phi=\varphi, \quad \Theta=\theta
$$

where $(R, \Phi, \Theta)$ and $(r, \phi, \theta)$ are the Euler and Lagrange spherical coordinates, respectively; and $c$ is the deformation parameter. Since the volume $V$ of the liquid diffusing in the hollow is a control parameter, the parameter $c$ in this study is given by the formula $c=3 / 4 \pi(V-V)$, where $V$ is the hollow volume before deformation. Thus, a hard loading of the shell is realized in this problem in the class of radial-symmetric deformations, whereby displacements are set on the hollow surface.

The equilibrium equations and the boundary conditions can be written as

$$
\begin{gathered}
\frac{d D_{r r}}{d r}+2 \frac{D_{r r}-D_{\theta \theta}}{r}=0,\left.\quad D_{r r}\right|_{r=b}=-p \frac{B^{2}}{b^{2}}, \\
\left.D_{r r}\right|_{r=a}=-p \frac{i A^{2}}{a^{2}},
\end{gathered}
$$

where $D_{r r}=2 \mu-\left(p R^{2} / r^{2}\right), D_{\theta \theta}=D_{\varphi \varphi}=2 \mu-(p r / R)$ are the diagonal components of the Piola stress tensor in the $\left(\mathbf{e}_{r}, \mathbf{e}_{\theta}, \mathbf{e}_{\varphi}\right)$ basis related to the Lagrange spherical coordinates; $A=R(a) ; B=R(b) ; a$ and $b$ are the internal and external radii of the shell before deformation, respectively; and $p^{\mathrm{e}}$ and $p^{\mathrm{i}}$ are the external and internal pressures acting on the shell, respectively. From (1) and (2), it follows that $p$ does not depend on $r$ and is determined by the relations

$$
p=p^{\mathrm{e}}-2 \mu \frac{b^{2}}{B^{2}}=p^{\mathrm{i}}-2 \mu \frac{a^{2}}{A^{2}},
$$

which yields the following relation for the internal pressure:

$$
p^{\mathrm{i}}=p^{\mathrm{e}}+2 \mu\left(\frac{b^{2}}{B^{2}}-\frac{a^{2}}{A^{2}}\right) .
$$

In what follows, the external pressure $p^{\mathrm{e}}$ is assumed to be zero (and the index "i" at the pressure is omitted).

Figure 1 shows the deformation (expansion) diagrams plotted as the internal pressure versus the internal shell surface displacement for various values of the shell thickness $h=(b-a) / b$. It can be shown that, for a spherical thick-walled shell, a descending region is also observed for other commonly used models of rubberlike materials (such as the Treloar material, MooneyRivlin media, Ogden materials at certain values of elastic constants, etc.). 
Instability. The stability of equilibrium of a nonlinearly elastic body (under conservative external loading conditions) is conventionally investigated using the Euler static method. This method is based on an analysis of equilibrium states slightly differing from the given state, with determination of the values of loading parameters under which the existence of nontrivial solutions to the equations of equilibrium with appropriate boundary conditions (linearized in the vicinity of the given state) is possible.

The linearized boundary-value problem for a sphere in the geometry of a reference configuration and in the absence of mass forces has the following form [13, 14]:

$$
\begin{gathered}
\operatorname{div} \mathbf{D}^{\cdot}=0,\left.\quad \mathbf{n} \cdot \mathbf{D}^{\cdot}\right|_{r=a}=p^{\mathrm{i}} \mathbf{n} \cdot \mathbf{C}_{0}^{-T} \cdot(\operatorname{grad} \mathbf{w})^{T} \cdot \mathbf{C}_{0}^{-T}, \\
\left.\mathbf{n} \cdot \mathbf{D} \cdot\right|_{r=b}=0,
\end{gathered}
$$

where the upper dots denote the linear increments of the corresponding parameters resulting from the superposition of small additional displacements $\mathbf{w}$ (for example, $\left.\mathbf{D}^{\cdot}=\left.\frac{d}{d \tau} \mathbf{D}\left(\mathbf{C}_{0}+\tau \operatorname{grad} \mathbf{w}\right)\right|_{\tau=0}\right)$, the subscript zero refers to the parameters calculated in the initial deformed state, and $\mathbf{n}$ is the normal to the shell surface.

Consider the axisymmetric perturbations

$$
\mathbf{w}=u \mathbf{e}_{r}+v \mathbf{e}_{\theta},
$$

and assume that the volume of the hollow filled by the liquid remains unchanged, which imposes the integral constraint on $\mathbf{w}$ :

$$
\left.2 \pi \int_{-\pi / 2}^{\pi / 2} u\right|_{r=a} \cos \theta d \theta=0 .
$$

In addition, these perturbations do not alter the pressure in the liquid filling the hollow, which is taken into account in boundary conditions (3).

Isoperimetric condition (5) excludes radially symmetric perturbations from consideration. Thus, considering the axisymmetric perturbations, we deal with the loading conditions intermediate between hard loading (when fixed displacements on the inner shell surface are set) and soft loading (when the hydrostatic pressure is set). In the former case, the stability of the shell is not lost. In the latter case, the shell becomes unstable with respect to the radially symmetric perturbations when the maximum on the deformation curve is reached (Fig. 1).
For the Bartenev-Khazanovich type material, the linearized Piola stress tensor has the following form [15]:

$$
\begin{gathered}
\mathbf{D}^{\cdot}=2 \mu \mathbf{A}^{\cdot}-p \cdot \mathbf{C}_{0}^{-T}+p \mathbf{C}_{0}^{-T} \cdot(\operatorname{grad} \mathbf{w})^{T} \cdot \mathbf{C}_{0}^{-T}, \\
\mathbf{A}^{\cdot}=\chi\left(\mathbf{e}_{r} \otimes \mathbf{e}_{\theta}-\mathbf{e}_{\theta} \otimes \mathbf{e}_{r}\right), \\
\chi=\left(\frac{r^{2}}{R^{2}}+\frac{R}{r}\right)^{-1}\left(\frac{\partial V}{\partial r}-\frac{1}{r}\left(\frac{\partial u}{\partial \theta}-v\right)\right) .
\end{gathered}
$$

The transformation of linearized equilibrium equations (3), the boundary conditions, and the linearized condition of incompressibility with allowance for expressions (4) and (6) leads to the system of equations

$$
\begin{gathered}
-\frac{R^{2}}{r^{2}} \frac{\partial p}{\partial r}+2 \mu \frac{1}{r}\left(\chi \tan \theta-\frac{\partial \chi}{\partial \theta}\right)=0 \\
-\frac{1}{R} \frac{\partial p}{\partial \theta}+2 \mu\left(\frac{\partial \chi}{\partial r}+\frac{\chi}{r}\right)=0 \\
\frac{R^{2}}{r^{2}} \frac{\partial u}{\partial r}+\frac{1}{R}\left(\frac{\partial v}{\partial \theta}-v \tan \theta+2 u\right)=0 \\
\frac{\partial v}{\partial r}-\frac{1}{r}\left(\frac{\partial u}{\partial \theta}-v\right)-\left(\frac{r^{2}}{R^{2}}+\frac{R}{r}\right) \chi=0
\end{gathered}
$$

and the boundary conditions (at $r=a$ and $r=b$ )

$$
-p^{\cdot}+2 \mu \frac{\partial u}{\partial r}=0, \quad \chi+\frac{1}{R}\left(\frac{\partial u}{\partial \theta}-v\right)=0 .
$$

A solution to the boundary-value problem (7)-(10) is found in the form of expansion in terms of the Legendre polynomials

$$
\begin{gathered}
u=\sum_{n=2}^{\infty} u_{n}(r) P_{n}(\sin \theta), \quad v=\sum_{n=2}^{\infty} V_{n}(r) P_{n}^{\prime}(\sin \theta) \cos \theta \\
p^{\cdot}=\sum_{n=2}^{\infty} p_{n}(r) P_{n}(\sin \theta) .
\end{gathered}
$$

The value of $n=1$ corresponds to the motion of the sphere as a rigid body and is not considered below. It can be seen from solution (11) that, by virtue of the orthogonality of the Legendre polynomials, the volume of the hollow inside the shell, which is filled by an incompressible liquid, remains unchanged. Therefore, condition (5) is fulfilled automatically. Substituting expression (11) into equations (7)-(10), we arrive at a linear homogeneous boundary-value problem for the system of ordinary differential equations for $u_{n}, v_{n}$, and $p_{n}$. 


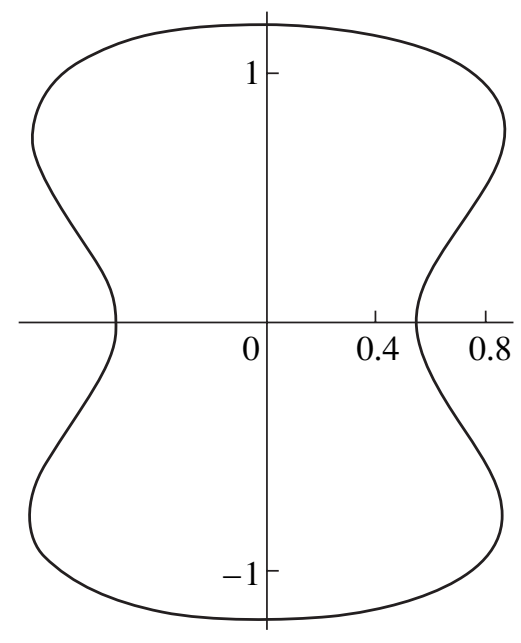

Fig. 2. The form of the loss of the spherical shape stability for $n=2$.

The critical values of the internal pressure were found numerically under the condition of nontrivial solvability of this boundary-value problem. In Fig. 1, the corresponding values are marked by dots on the deformation diagrams. As can be seen, these dots belong to the dropping section of the diagram, by analogy with the results obtained for the beam tension [4] and the cylinder expansion $[5,6]$. We have calculated the number $n$ of the expansion mode corresponding to the instability. It turned out that the mode appearing first always corresponds to $n=2$. As can be seen from Fig. 1, the first bifurcation point is rather distant from the point of maximum in the diagram. This means that the stress-strain state of the shell, corresponding to the initial region in the descending branch of the deformation diagram, is locally stable. The forms of the instability are shown in Fig. 2.

Thus, the nonspherical shape of the initially spherical particles can result from the instability during expansion. Note that deformed spheres having a shape similar to that shown in Fig. 2 were observed in experiment [2]. The instability can be avoided if the polymer shell is made of a material whose deformation diagrams have no descending regions or if we can exclude the system passage to such a descending region.

\section{ACKNOWLEDGMENTS}

This study was supported in part by the Russian Foundation for Basic Research (project no. 05-01-00638); the Russian Science Support Foundation; and the Basic Research Program of the Department of Power Engineering, Machine Building, Mechanics, and Control Processes of the Russian Academy of Sciences.

\section{REFERENCES}

1. V. N. Pavlyuchenko, Vysokomol. Soedin., Ser. C 46, 2140 (2004).

2. V. N. Pavlyuchenko, O. V. Sorochinskaya, S. S. Ivanchev, et al., J. Polym. Sci., Part A: Polym. Chem. 39, 1435 (2001).

3. V. N. Pavlyuchenko, O. V. Sorochinskaya, O. N. Primachenko, et al., J. Polym. Sci., Part A: Polym. Chem. 42, 2225 (2004).

4. L. M. Zubov and A. N. Rudev, Prikl. Mat. Mekh. 60, 786 (1996).

5. L. M. Zubov and M. S. Lastenko, Izv. Ross. Akad. Nauk, Mekh. Tverd. Tela, No. 3, 135 (2004).

6. L. M. Zubov and D. N. Sheĭdakov, Prikl. Mat. Mekh. 69, 53 (2005).

7. V. A. Ibragimov and V. D. Klyushnikov, Izv. Akad. Nauk SSSR, Mekh. Tverd. Tela, No. 4, 116 (1971).

8. L. V. Nikitin and E. I. Ryzhak, Izv. Akad. Nauk SSSR, Mekh. Tverd. Tela, No. 2, 155 (1986).

9. V. I. Feodos'ev, Prikl. Mat. Mekh., No. 2, 339 (1968).

10. Ya. G. Panovko and I. I. Gubanova, Stability and Oscillations of Elastic System (Nauka, Moscow, 1979) [in Russian].

11. N. F. Morozov, Izv. Vyssh. Uchebn. Zaved., Mat. 1 (2), 127 (1961).

12. N. V. Banichuk, Optimization of Forms of Elastic Bodies (Nauka, Moscow, 1980) [in Russian].

13. A. I. Lur'e, Nonlinear Theory of Elasticity (Nauka, Moscow, 1980) [in Russian].

14. A. I. Lur'e, Theory of Elasticity (Nauka, Moscow, 1970) [in Russian].

15. V. A. Eremeyev, Izv. Sev.-Kavk. Nauchn. Tsentra Vyssh. Shk., Estestv. Nauki, No. 1, 55 (1990).

Translated by Yu. Vishnyakov 